\section{Recovery of raptors from displacement by wind farms - a response}

Dohm et al. (2019) describe temporal trends in the displacement of resident raptors at a single wind farm. They report the recovery of overall raptor abundances 7-8 years post-construction, although with varying displacement trends among species (or species/groups), and this finding is used to suggest that displacement of raptors caused by wind farms may be temporary. They also state that "In the absence of longer-term monitoring, our findings may be broadly applicable to the US wind industry". Even though this study provides valuable long-term data on raptor displacement by wind farms, we found weaknesses in its analysis and conclusions that warrant further discussion.

Dohm et al.'s findings provide a limited representation of raptor displacement by wind farms, given that they refer to only one wind farm, reflecting impacts of a small number of wind turbines in a small area; lack data from winter and migration periods; and include only five cases (four species and one species/ group) among which only three showed patterns of recovery. Among those cases, turkey vultures (Cathartes aura) exhibited the most prominent recovery, with abundances 7-8 years post-construction being even higher than during the preconstruction phase. The observed increase in abundance of turkey vulture - an obligate scavenger - may have been caused by its attraction to the carcasses of birds killed by collision with turbines (Grodsky et al. 2013). This species is likely driving the trend of recovery observed for all species combined (Figure 1 in Dohm et al. [2019]), as it represents nearly $60 \%$ of the birds counted in 2015 and 2016. Mixed results were associated with the two accipiter species, where 2016 (but not 2015) postconstruction abundance was similar to pre-construction abundance.

Dohm et al.'s results are likely based on resightings of a small number of individual birds. Raptors in general demon- strate a high degree of site fidelity, particularly during the breeding season (when most data were collected), and different individuals (or pairs) tend to avoid territory overlap to reduce food competition (Newton 1979). The focal species have home ranges varying from 1 to $25 \mathrm{~km}^{2}$ (Newton 1979; Andersen and Rongstad 1989; Dechant et al. 2002; Reynolds 2002; Holland et al. 2017); consequently, only a few non-overlapping home ranges would fit in the study area (approximately $131 \mathrm{~km}^{2}$ ). Dohm et al. reported 477 counts from 12 sites, approximately 40 repeated counts per site (assuming equal distribution). Therefore, we expect large redundancy in the data of this study, with counts being more representative of raptor flight activity than of population trends. Even though the authors seem to have correctly controlled for the different data dependence levels in their models, doing so does not counteract their small sample sizes for each species.

Dohm et al. may have also inappropriately evaluated displacement. Previous research has documented that displacement occurs up to a distance of some hundreds of meters from each turbine. Two studies on raptors reported a displacement distance up to 700 and 800 meters (Pearce-Higgins et al. 2009; Marques et al. 2019). However, in Dohm et al.'s article, five of the 12 survey sites were located at distances $>1 \mathrm{~km}$ from any turbine, where the birds are probably unaffected by turbine presence (PearceHiggins et al. 2009; Marques et al. 2019). In addition, because Dohm et al.'s data are sufficient for evaluating the effect of the turbine proximity on bird abundances (as per Pearce-Higgins et al. [2009] and Marques et al. [2019]), demonstrating that this effect diminishes with time would have provided stronger evidence that raptor displacement declines in the long term than the results currently presented.

Although we agree with Dohm et al. that there is a lack of published results on long-term displacement effects on raptors by wind farms, we think the authors did not adequately draw attention to the available studies whose main findings contradict their own. Hunt et al. (1995), which was not cited by Dohm et al., reported declines of golden eagle (Aquila chrysaetos) densities 12 years after the construction of a wind farm. Stewart et al. (2007) provided a meta-analysis with strong evidence for long-term declines of birds (including raptors) near wind turbines; while this study is cited by Dohm et al., its results could have been discussed in further detail. The results from Marques et al. (2019) also support the long-term prevalence of raptor displacement by wind farms, in this case 10 years (or more) post-construction for black kites (Milvus migrans), although the post-construction time is not provided in their published article.

For these reasons, we think that Dohm et al.'s conclusions are overstated and could be misused by the proponents of the wind energy industry as an excuse to disregard or downplay the facilities' negative impacts on raptors. We stress that site- and species-specific findings cannot be applied to the wind industry in general. However, we agree with Dohm et al. (2019) that regulatory agencies should encourage developers to conduct long-term monitoring of raptor displacement by wind turbines using standardized methods, which would allow the analysis of commonalities and variability across species and contexts in the future.

\section{Carlos David Santos ${ }^{1,2^{*}}$, Ana Teresa Marques $^{3,4}$, and Roel May ${ }^{5}$ \\ ${ }^{1}$ Núcleo de Teoria e Pesquisa do Comportamento, Universidade Federal do Pará, Belém, Brazil; ${ }^{2}$ Department of Migration, Max Planck Institute for Animal Behavior, Radolfzell, Germany *(cdsantos@ab.mpg.de); ${ }^{3}$ cE3c - Centro de Ecologia, Evolução e Alterações} Ambientais, Faculdade de Ciências, Universidade de Lisboa, Lisboa, Portugal; ${ }^{4} \mathrm{CIBIO} / \mathrm{InBIO}$, Centro de Investigação em Biodiversidade e Recursos Genéticos, Laboratório Associado, Universidade do Porto, Campus Agrário de Vairão, Vairão, Portugal; ${ }^{5}$ Norwegian Institute for Nature Research, Terrestrial Ecology Department, Trondheim, Norway 
Andersen DE and Rongstad OJ. 1989. Homerange estimates of red-tailed hawks based on random and systematic relocations. $J$ Wildl Manag 53: 802-07.

Dechant JA, Sondreal ML, Johnson DH, et al. 2002. Effects of management practices on grassland birds: northern harrier. Jamestown, ND: USGS Northern Prairie Wildlife Research Center.

Dohm R, Jennelle CS, Garvin JC, et al. 2019. A long-term assessment of raptor displacement at a wind farm. Front Ecol Environ 17: 433-38.

Grodsky SM, Jennelle CS, and Drake D. 2013. Bird mortality at a wind-energy facility near a wetland of international importance. Condor 115: 700-11.

Holland AE, Byrne ME, Bryan AL, et al. 2017. Fine-scale assessment of home ranges and activity patterns for resident black vultures (Coragyps atratus) and turkey vultures (Cathartes aura). PLoS ONE 12: e0179819.

Hunt G, Walton B, Drager T, et al. 1995. A pilot golden eagle population study in the Altamont Pass Wind Resource Area, California. Santa Cruz, CA: Predatory Bird Research Group, University of California.

Marques AT, Santos CD, Hanssen F, et al. 2019. Wind turbines cause functional habitat loss for migratory soaring birds. J Anim Ecol; doi.org/10.1111/1365-2656.12961.

Newton I. 1979. Population ecology of raptors. Berkhamsted, UK: T \& AD Poyser.

Pearce-Higgins JW, Stephen L, Langston RHW, et al. 2009. The distribution of breeding birds around upland wind farms. J Appl Ecol 46: 1323-31.

Reynolds RT. 2002. Management of western coniferous forest habitat for nesting accipiter hawks. Fort Collins, CO: USDA Forest Service.

Stewart GB, Pullin AS, and Coles CF. 2007. Poor evidence-base for assessment of windfarm impacts on birds. Environ Conserv 34: 1-11.

\section{Documented raptor displacement at a wind farm}

In their letter, Santos et al. describe several perceived weaknesses of our study (Dohm et al. 2019). We appreciate the opportunity to respond to Santos et al's criticisms, which primarily relate to the strength and applications of our conclusions based on our study design, data analysis, and review of available literature.

Regarding experimental design and data analysis, Santos et al. express concerns that we examined only one wind facility, that we lacked data on raptors during winter and migratory periods, and that our results were likely based on resightings of only a small number of individual birds. While we share their reservations regarding the lack of site replication, we do not agree with their other assertions.

In our original article, we clearly stated our decision to limit analysis of our pre- and post-construction surveys to the late spring and summer, to avoid raptor migration and to focus on raptors resident to the study area. We contend that our focus on a seasonal subset does not weaken our conclusions and that our long-term data are valuable for understanding the wind industry's impact on raptors.

Furthermore, while we recognize that resightings of the same (previously observed) individuals likely influenced our metric of raptor abundance, we have no reason to suspect that resighting rates varied temporally for the species we analyzed. We also acknowledged that the metric of activity was a proxy for abundance as opposed to a measure of true abundance, which refutes one of Santos et al's chief concerns: that we interpreted our findings to be representative of raptor populations. On the contrary, we explicitly stated that "Our research objective was to investigate the duration of wind farm displacement...by comparing raptor abundance", and that our analysis was limited to raptor abundances within the immediate vicinity of the wind farm. We only investigated population trends statewide using Breeding Bird Survey data as a control to determine whether the abundance fluctuations observed at the wind farm were different relative to fluctuations in areas outside the wind farm.

Santos et al. also suggest that observed increases in turkey vultures (Cathartes aura) may have driven the observed recovery of pooled raptor abundance in the long term. However, we analyzed all species/groups in a series of separate, predictive models. While our analysis of all raptors combined was likely influenced by turkey vultures, this would not account for the positive trends described for redtailed hawks (Buteo jamaicensis) and for the accipiter (Accipiter spp) group, two analyses conducted independently of the turkey vulture observations.

Regarding their suggestion that we inappropriately evaluated displacement, we recognize that there are multiple ways of defining displacement and assessing its occurrence. For this reason, we specifically defined our use of the term displacement as "the reduced density of birds occurring within or immediately adjacent to wind-power plants due to long-term disturbance leading to functional habitat loss" (May 2015). We are confident that our study design - with survey locations throughout the wind farm - was appropriate for measuring this type of displacement at the scale of a single facility.

We appreciate Santos et al. bringing the work by Marques et al. (2019) and Hunt et al. (1995) to our attention. The former reference focused on migratory raptors set in a very different landscape, making comparisons to our study challenging. In the case of the latter reference, we believe that Hunt et al's (1995) analysis of turbine-related mortality of golden eagles was inappropriate for comparison with our study, given that high levels of raptor mortality were not documented at our study site (Grodsky et al. 2013). Furthermore, the Altamont Pass Wind Resource Area, Hunt et al's (1995) study site, is a very different wind facility as compared to the modern wind facility we studied.

Finally, we disagree with Santos et al. that our conclusions are overstated and likely to be used by the wind industry to disregard the impacts of wind facilities on raptors. We provide ample caveats on our study's limitations as well as the limitations of typical raptor studies performed at wind facilities in the US, and we also emphasize the need for additional longterm studies. Furthermore, we warn that the cumulative impacts of disturbance could be substantial, regardless of whether displacement impacts at an individual 